INFLAMMATORY BOWEL DISEASE

\title{
Mucosal colonisation with Lactobacillus casei mitigates barrier injury induced by exposure to trinitronbenzene sulphonic acid
}

\author{
M Llopis, M Antolín, F Guarner, A Salas, J-R Malagelada
}

Gut 2005;54:955-959. doi: 10.1136/gut.2004.056101

Background: Trinitrobenzene sulphonic acid (TNBS) induces chronic transmural inflammatory lesions in the rat colon. Injury is facilitated by barrier disruption and invasion of commensal bacteria. However, certain bacteria have shown anti-inflammatory properties in in vitro models.

See end of article for authors' affiliations

Correspondence to: Dr F Guarner, Digestive System Research Unit, Hospital General Vall d'Hebron, Barcelona 08035, Spain; fguarnera@medynet.com

Revised version received 28 October 2004

Accepted for publication

9 November 2004
Aim: To investigate in vivo the anti-inflammatory effect of Lactobacillus casei DN-114001.

Methods: Rats with a colonic segment excluded from faecal transit were surgically prepared. After washing the lumen with antibiotics, the excluded segment was recolonised (control group: standard flora of rat origin; test group: standard flora and $L$ casei). Microbial colonisation was confirmed by culture of segment washing, and colitis was then induced by instillation of TNBS. One day after, intestinal lesions were blindly graded by macro- and microscopic scores, and myeloperoxidase activity measured in tissue homogenates. Translocation of bacteria to mesenteric lymph nodes, spleen and liver was investigated. Results: Test rats showed a smaller area of mucosal injury than control rats $(p<0.05)$. Maximum depth lesion scores were similar in both groups but myeloperoxidase activity was lower in test than in control rats $(p<0.05)$. Remarkably, bacterial translocation was quantitatively lower $(p<0.01)$ and less frequent $(p<0.05)$ in test than in control rats.

Conclusion: In rats colonised with $L$ casei, mucosal injury, inflammatory response, and barrier disruption after TNBS challenge were attenuated. Bacterial communities colonising the mucosa can modify inflammatory responses to luminal challenges.
$\mathrm{T}$ he trinitrobenzene sulphonic acid (TNBS) model of experimental colitis in the rat is characterised by chronic inflammatory lesions that are induced by a single intracolonic application of TNBS in diluted ethanol. ${ }^{1}$ Typical TNBS induced lesions may persist for up to 6-8 weeks, and consist of chronic mucosal ulcerations with underlying inflammatory cell infiltrate that may involve the whole intestinal wall through to the serosa. Bowel strictures and serosal adhesion to surrounding tissues are common features of this model of intestinal inflammation. Interestingly, transmural inflammation is not observed in other animal models of colitis induced by exposure to a chemical. ${ }^{23}$

Several studies have investigated the mechanism by which TNBS induces chronic transmural inflammation. TNBS can modify cell surface proteins by forming covalent bonds with lysine groups, and acts as a hapten that induces immune responses to the modified proteins. ${ }^{4}$ Moreover, macrophage mediated cytotoxicity against TNBS modified cells has been described in vitro. ${ }^{5}$ Immune mechanisms involved in delayed hypersensitivity reactions to the hapten could explain the progression of TNBS induced intestinal lesions to chronicity. ${ }^{1}$ However, it has also been shown that TNBS deteriorates mucosal barrier function by interacting with surface active phospholipids of the colonic mucosa. ${ }^{6}$ Shortly after intracolonic administration, TNBS reduces surface hydrophobicity and increases the tissue susceptibility to bacteria and water soluble noxious agents. ${ }^{6}$ In fact, TNBS by itself exerts direct toxicity on intestinal epithelial cell monolayers without intervention of macrophages. ${ }^{3}$ The direct effect is abrupt and results in epithelial cell necrosis. Thus in this model, disruption of the surface barrier and direct toxicity of TNBS on epithelial cells appear to be the initiating events leading to intestinal inflammation.
Previous studies from our laboratory demonstrated that several bacterial species of the common rat microflora, including anaerobes, invade the colonic wall after disruption of the epithelium by TNBS. ${ }^{7}$ Viable translocated bacteria can be observed and recovered from the colonic wall as soon as 24 hours after TNBS challenge. Using an in vivo model of controlled microbial colonisation (colonic segment excluded from faecal transit), we found that luminal bacteria play a crucial role in induction of the inflammatory response to TNBS. $^{7}$ After radical eradication of the indigenous flora by antibiotics, no significant mucosal inflammation was observed in response to intracolonic instillation of the hapten. A full response to TNBS, as manifested by deep transmural inflammatory lesions, was only observed in rats colonised with certain anaerobes, such as some species of Bacteroides and Clostridium genera. ${ }^{7}$

The aim of the present study was to investigate whether some bacteria may downregulate the inflammatory response induced by TNBS. Based on previous observations obtained by in vitro experiments, ${ }^{8} 9$ we hypothesised that some bacteria mitigate the inflammatory response to luminal challenges. In organ culture experiments, Lactobacillus casei was shown to reduce spontaneous release of tumour necrosis factor $\alpha$ by inflamed intestinal tissue, and also the cytokine response induced by Escherichia coli. ${ }^{8}$ In this study, we determined whether mucosal colonisation with the $L$ casei strain also exerts an anti-inflammatory effect in an in vivo setting.

Abbreviations: TNBS, trinitrobenzene sulphonic acid; MPO, myeloperoxidase; CFU, colony forming units; MLN, mesenteric lymph nodes; MRS, De Man Rogosa Sharp medium 


\section{METHODS}

Animals

Male Sprague-Dawley rats weighing 225-250 g were purchased from Charles River Laboratories (St Germain sur l'Arbresle, Lyon, France). Animals were free of all pathogens listed in the FELASA guidelines ${ }^{10}$ and were housed under standard conditions (room temperature $22^{\circ} \mathrm{C}$, humidity $50 \pm 5 \%, 12 / 12 \mathrm{~h}$ light-dark cycle). All experiments were conducted in accordance with the National Insitutes of Health Guide for the Care and Use of Laboratory Animals (DHEW Publication No (NIH) 85-23, revised 1985).

Rats were weighed on days 1, 2, 3, 6, and 7 after surgery and controlled for the indicative pain, distress, and discomfort behaviour, ${ }^{11}$ to apply appropriate end points when necessary (Guide for the Care and Use of Laboratory Animals, National Academy Press, Washington DC, 1996).

\section{Experimental model}

Rats with a colonic segment excluded from faecal transit were surgically prepared as previously described by Favia and colleagues $^{12}$ Briefly, a colonic segment is brought out to the anterior abdominal wall through two colostomies, and continuity of the intestinal tract is achieved by anastomosis of the terminal ileum to the distal colon. This model obtains a suitable in vivo ecological environment for controlled mucosal colonisation with selected bacterial strains after elimination of the native flora by non-absorbable broad spectrum antibiotics. ${ }^{7}$

\section{Experimental design}

Rats with an excluded colonic segment were randomly distributed into three groups. The control group consisted of 16 rats and the test group 17 rats. A blank group $(n=4)$ was conducted in parallel and included rats not subjected to TNBS instillation. One day after surgery, rats were lightly anaesthetised $(5 \mathrm{mg} / \mathrm{kg}$ ketamine (Parke-Davis, Ann-Arbor, Michigan, USA) and $1 \mathrm{mg} / \mathrm{kg}$ xylazine (Bayer, Leverkusen, Germany)) and the lumen of the excluded segment was thoroughly flushed with a solution of broad spectrum antibiotics ( $50 \mathrm{mg} / \mathrm{ml}$ imipenem and $50 \mathrm{mg} / \mathrm{ml}$ vancomycin) that was kept in the lumen for three hours. Thereafter, the lumen was washed with $60 \mathrm{ml}$ of sterile saline. Then, $3 \mathrm{ml}$ of a suspension of aerobic bacteria were inoculated into the segment lumen. The suspension was prepared as described below and included Enterobacter aerogenes, Klebsiella pneumoniae, and Streptococcus viridans, with (test group) or without (control and blank groups) Lactobacillus casei strain DN-114 001 (Danone-Vitapole, Palaiseau, France). Colostomies were closed by ligature immediately after inoculation.

On day 3, colostomies were reopened and the colonic lumen was washed with $10 \mathrm{ml}$ of a Schaedler reduction medium (Bio Mérieux, Marcy l'Etoile, France). Mucin aggregates in the lumen were removed. Thereafter, $3 \mathrm{ml}$ of a liquid suspension of anaerobic bacteria were inoculated. The suspension was prepared as described below and included Bacteroides fragilis and Bacteroides disastonis, with (test group) or without (control and blank groups) the $L$ casei strain. Colostomies were immediately closed by ligature.

On day 6 after surgery, the presence of viable bacteria in the segment was controlled by microbiological culture of colostomy effluent. The effluent was obtained under light anaesthesia (ketamine-xylacine) by flushing the lumen segment with up to $10 \mathrm{ml}$ of Schaedler reduction medium (mucin aggregates were removed). Aliquots ( $1 \mathrm{ml}$ ) of the effluent were carefully collected in sterile plastic syringes, avoiding contact with air. They were immediately processed for microbiological culture. Thereafter, colitis was induced in control and test rats by intraluminal instillation of $3 \mathrm{ml}$ of TNBS solution $(45 \mathrm{mg} / \mathrm{ml}$ in $10 \%$ ethanol) (TNBS from
Sigma, St Louis, Missouri, USA and ethanol from Panreac Barcelona, Spain).

On day 7 after surgery, rats were anaesthetised with ketamine-xylacine and subjected to laparotomy. Mesenteric lymph nodes, the spleen, and the left hepatic lobe were carefully removed under sterile conditions for later investigation of bacterial translocation. Euthanasia was performed by exsanguination and the colonic segment was removed and divided into two parts by a longitudinal section. One specimen was fixed in $4 \%$ paraformaldehyde (Panreac) and processed for histological assessment of tissue lesions. The second specimen was stored at $-20^{\circ} \mathrm{C}$ for later myeloperoxidase assay (MPO).

\section{Inocula}

Bacterial suspensions for inoculation were prepared from cultures of each individual species. All bacterial species used in this protocol except for the $L$ casei strain were isolated from the rat, as previously described. ${ }^{7}$ The $L$ casei strain was provided by Danone-Vitapole (Palaiseau, France). Aerobic bacteria were grown in liquid culture medium at $37^{\circ} \mathrm{C}$ under a controlled atmosphere $\left(5 \% \mathrm{CO}_{2}\right): L$ casei and $S$ viridans were grown in De Man Rogosa Sharp medium (MRS; Difco, Detroit, Michigan, USA), and $E$ aerogenes and $K$ pneumoniae were grown in a $1 \%$ tryptone peptone medium (Difco), $0.5 \%$ $\mathrm{NaCl}$, and $0.5 \%$ yeast extract (Difco). $B$ fragilis and $B$ disastonis were grown in Schaedler medium (Difco) for 48 hours at $37^{\circ} \mathrm{C}$ in an anaerobic atmosphere (Anaerocult A Merck, Darmstadt, Germany). At the end of the phase of exponential growth, bacterial cultures were stopped. The final aerobic inoculum concentration for each control and test group rat was $6 \times 10^{9}$ colony forming units $(\mathrm{CFU}) / \mathrm{ml}$. In the control group, $1 \mathrm{ml}$ of a $6 \times 10^{9} \mathrm{CFU} / \mathrm{ml}$ bacterial culture for each of the three bacteria (see above) were mixed, with a final volume of $3 \mathrm{ml}$. In the test group, $0.75 \mathrm{ml}$ of a $6 \times 10^{9} \mathrm{CFU} / \mathrm{ml}$ bacterial culture for each of the four bacteria (see above) were mixed, again with a final volume of $3 \mathrm{ml}$. The final anaerobic inoculum concentration for each control and test group rat was $5 \times 10^{9} \mathrm{CFU} / \mathrm{ml}$. In the control group, $1.5 \mathrm{ml}$ of a $5 \times 10^{9} \mathrm{CFU} / \mathrm{ml}$ bacterial culture for each of the two bacteria (see above) were mixed, with a final volume of $3 \mathrm{ml}$. In the test group, $1 \mathrm{ml}$ of a $5 \times 10^{9} \mathrm{CFU} / \mathrm{ml}$ bacterial culture for each of the three bacteria (see above) were mixed, again with a final volume of $3 \mathrm{ml}$. Volumes of $3 \mathrm{ml}$ were instilled into the lumen of each colonic segment.

\section{Microbiological studies}

Samples of washings were collected under sterile conditions before laparotomy. Serial decimal dilutions (from log -2 to -8 ) were performed in Schaedler reduction medium. Petri dishes of various selective media were inoculated and incubated. Enterobacteria and enterococci were selected in trypcase soya agar (Bio Mérieux) $5 \%$ blood at $37^{\circ} \mathrm{C}$ for 48 hours in a microaerobic atmosphere $\left(5 \% \mathrm{CO}_{2}\right)$ and later differentiated in Tergitol 7 Agar TTC (Reactivos para Diagnostico, Barcelona, Spain), Brilliant Green Bile Agar (Difco), and Eosin Methylene Blue Agar (Bio Mérieux) under the same conditions for colony colour and morphology. Bacteroides were selected in Schaedler Neo-Vanco (Bio Mérieux) for 48 hours at $37^{\circ} \mathrm{C}$ in an anaerobic atmosphere (Anaerocult A; Merck) and later verification was assessed by growth in BBE Amikacin (Biomedics, Madrid, Spain) and lack of growth in an aerobic atmosphere. $L$ casei was differentiated in MRS (Difco) at $37^{\circ} \mathrm{C}$ for 72 hours in a microaerobic atmosphere $\left(5 \% \mathrm{CO}_{2}\right)$ and selected for colony colour and morphology. After incubation, the colonies were counted and further identified by biochemical methods (gram staining, catalase, and oxidase) and by API $50 \mathrm{CH}$ gallery for lactobacilli, API 10S for enterobacteria, and API 
Table 1 Quantitative analysis of microbial isolates in lumen washings of excluded colonic segments

\begin{tabular}{lllr}
\hline & Control group & Test group & p Value \\
\hline Inoculated Enterobacteriae & $5.4(2.3-7.3)$ & $4.3(2.3-7.6)$ & 0.59 \\
Inoculated Streptococcus & $5.9($ nd-7.6) & $5.3(3.6-5.7)$ & 0.96 \\
Inoculated Bacteroides & $3.3(2.3-5.7)$ & $6.0(4.4-7.6)$ & 0.15 \\
Inoculated L casei & nd & $3.6(2.9-6.6)$ & $<0.01$ \\
Non-inoculated bacteria & $5.3($ nd-7.2) & $5.3($ nd -7.3$)$ & 0.99 \\
Total bacteria & $6.1(2.6-7.9)$ & $6.2(4.4-8.0)$ & 0.91 \\
\hline
\end{tabular}

nd, not detected; detection limit 10 colony forming units per washing.

Data are $\log _{10}$ of colony forming units per washing, expressed as median (25th-75th percentile) values.

Differences were tested using the Mann-Whitney U test.

20A (Bio Mérieux) for anaerobes. Results are expressed as $\log _{10}$ of CFU per washing sample.

\section{Bacterial translocation}

Detection of viable enteric bacteria in mesenteric lymph nodes (MLN) is considered the gold standard to evidence bacterial translocation. ${ }^{13}$ Samples of MLN, left hepatic lobe, and spleen were removed under sterile conditions before the death of the rat. Tissues were dissected free from fat, weighed, and divided into two equal parts. Samples of MLN, liver, or spleen were respectively homogenised in 3, 5, or $2 \mathrm{ml}$ of brain heart infusion (Difco) or Schaedler media (Bio Mérieux) for cultures of aerobic and anaerobic microorganisms, respectively. Serial decimal dilutions of the aliquots were plated in Petri dishes containing various selective media, as described above. After incubation, colonies were counted and identified as above. Results are expressed as $\log _{10}$ of CFU/g of tissue.

\section{Myeloperoxidase assay}

Colonic segments were homogenised in $2 \mathrm{ml}$ of phosphate buffered saline using a tissue tearor (model 985-370; Biospec, Racine, Wisconsin, USA) and centrifuged. The pellets were again homogenised in an equivalent volume of phosphate buffer $50 \mathrm{mM}$, pH 6.0, containing $0.5 \%$ hexadecyltrimethylammonium bromide (Sigma) and $5 \mathrm{mM}$ EDTA (Sigma), sonicated three times for 30 seconds (Labsonic 2000; Braun, Melsungen, Germany) and centrifuged. Supernatants were used for determination of tissue MPO activity by a kinetic method with o-dianisidine and $\mathrm{H}_{2} \mathrm{O}_{2}$. One unit of enzyme activity is defined as the amount of MPO that degrades $1 \mu \mathrm{mol}$ of peroxide per minute at $25^{\circ} \mathrm{C}$. $^{7}$

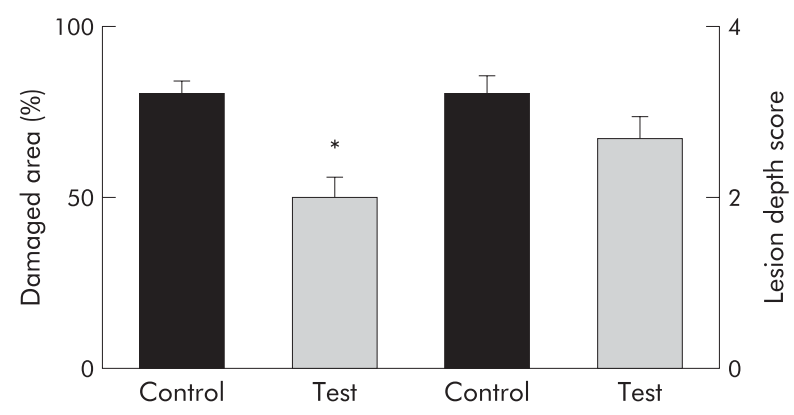

Figure 1 Lesion scores after induction of colitis by trinitrobenzene sulphonic acid in rats with an excluded colonic segment. In control rats the mucosal surface area affected by any grade of mucosal damage was significantly larger than that in test rats $\left({ }^{*} p<0.05\right)$. No significant difference was found in histological scores evaluating the maximum depth of the lesions.

\section{Histological studies}

A code was assigned to each colon. Two investigators, unaware of the treatment group, determined the extent of the damaged area as per cent of total mucosal surface in the excluded colonic segment. Sections of paraffin embedded colonic specimens were stained with haematoxylin-eosin. Two independent pathologists examined the coded tissue sections and scored the maximum depth of the lesions $(0$, normal histology; 1, oedema and mucosal congestion; 2, necrosis limited to the mucosa; 3 , necrosis in the mucosa and submucosa; 4, necrosis in the mucosa and submucosa with involvement of the muscularis propia; and 5, transmural necrosis). After examination of 6-10 sections per animal, the maximum depth lesion score was recorded.

\section{Statistical analysis}

Results are expressed as mean (SEM) or as median (range), when indicated. Significant differences between means were determined using the Student's $t$ test or the Mann-Whitney U test, as appropriate. Fisher's exact test was used for comparison of translocation rates.

\section{RESULTS}

\section{Colonisation of the excluded segment}

Bacterial species recovered from the excluded colonic segment in each of the two experimental groups, prior to induction of colitis by TNBS, are shown in table 1. Inoculated bacteria were recovered in both groups, confirming survival of the strains. Furthermore, total bacterial counts in segment washings were similar in both groups. No significant differences in individual isolates were found, except for $L$ case $i$ which was recovered at a median level of 3 logarithmic orders per washing in the test group but was not detected in any of the rats in the control group.

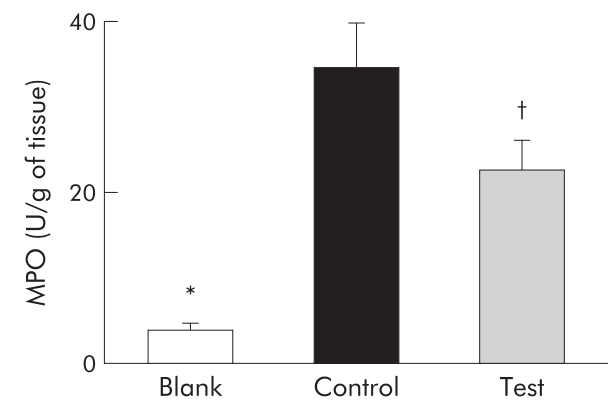

Figure 2 Myeloperoxidase (MPO) activity in homogenates from the excluded colonic segment. Data are shown for rats with control flora, not subjected to trinitrobenzene sulphonic acid (TNBS) instillation (Blank), for rats with control flora subjected to TNBS instillation (Control) ( ${ }^{*} p<0.05 \mathrm{v}$ blank), and for rats with control flora plus Lactobacillus casei subjected to TNBS instillation (Test) ( ${ }^{*} p<0.05 v$ blank; $\uparrow p<0.05 v$ control). 


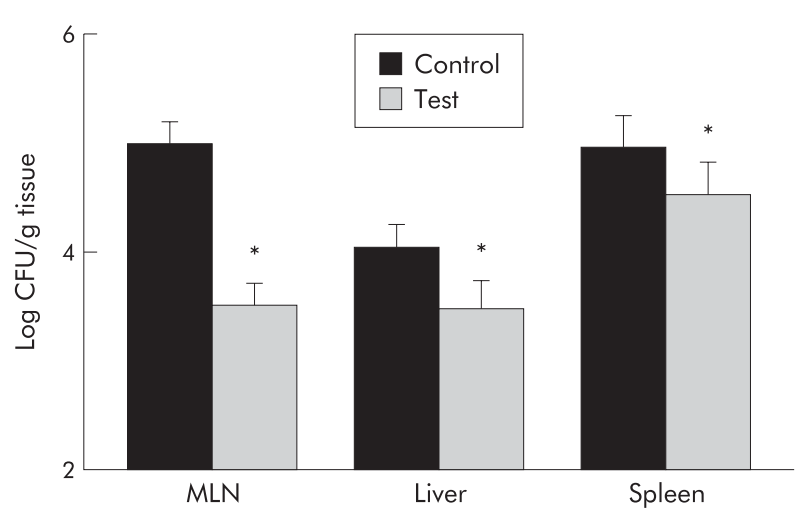

Figure 3 Quanitative recovery of viable bacteria translocated to extraintestinal sites after induction of trinitrobenzene sulphonic acid in control and test rats ( ${ }^{*} \mathrm{p}<0.05 \mathrm{v}$ control). CFU, colony forming units; $M L N$, mesenteric lymph nodes.

\section{Effect of bacteria on TNBS colitis}

Administration of TNBS into the excluded colonic segment induced severe mucosal lesions involving a large mucosal surface area in all rats. Most mucosal lesions were deep and involved the submucosa. The difference in maximum lesion depth scores between the control and test groups was not significant. However, the extent of mucosal surface affected by lesions was significantly different in both groups. As shown in fig 1 , the percentage of damaged mucosal area in rats colonised with the $L$. casei strain was significantly lower than that in control rats $(\mathrm{p}<0.05)$.

Figure 2 shows MPO activity in homogenates of the excluded colonic segment. In rats with TNBS induced colitis (control and test groups), MPO activity was higher than in rats not subjected to TNBS (blank group). Interestingly, test rats showed significantly lower tissue MPO activity than control rats $(\mathrm{p}<0.05)$.

\section{Bacterial translocation in TNBS colitis}

Bacterial translocation to distant organs was confirmed by microbiological culture techniques. Quantitative analysis of bacteria recovered in MLN, liver, and spleen of rats with TNBS induced colitis is shown in fig 3 . Recovery of bacteria was significantly lower in rats from the test group compared with controls $(\mathrm{p}<0.05)$.

In blank rats, translocation to MLN was assessed on day 6 after surgery with no induction of TNBS colitis. Positive microbial translocation due to surgery was detected in all rats, and bacterial recoveries ranged from $10^{3}$ to $10^{4} \mathrm{CFU} / \mathrm{g}$ of tissue. Considering this background level of bacterial translocation due to surgery, only excess translocation would be attributable to TNBS induced colitis. Recovery of bacteria in MLN above $10^{4} \mathrm{CFU} / \mathrm{g}$ of tissue was considered to be due to barrier disruption by TNBS. After induction of colitis, all rats in the control group presented excess bacterial translocation to MLN (above $10^{4} \mathrm{CFU}$ ) compared with none of the rats in the test group $(\mathrm{p}<0.05)$.

\section{DISCUSSION}

The rat model that we used in the present investigation, consisting of a colonic segment that was surgically excluded from faecal transit, allowed relatively effective control of in vivo microbial colonisation of the intestinal mucosa. Indeed, the selected bacterial strains that are introduced into the lumen of the segment spontaneously persisted for several days. The term colonisation classically describes the ability of a particular bacteria strain to permanently establish in the host over time, without the need for daily reintroduction of the bacteria. ${ }^{14}$ This requirement was fulfilled in the excluded segment of our model, in which lack of a pre-established indigenous community of bacteria facilitated implantation of the inoculated strains. Using this model, we have previously shown that invasion of bacteria into the intestinal wall is an essential step in the progression of TNBS induced injury towards transmural inflammation. ${ }^{7}$ The current study demonstrates that other bacterial strains colonising the intestinal lumen may significantly ameliorate TNBS induced intestinal lesions.

In rats colonised with the $L$ casei strain, the extent of injured colonic mucosa after exposure to TNBS was significantly smaller than in control rats. Although no differences were found in the maximum depth of the lesions, the mucosal inflammatory response, as assessed by leucocyte infiltration into the intestinal wall (tissue MPO activity), was also significantly reduced in rats colonised with $L$ casei. In addition, our data indicate that mucosal barrier disruption by TNBS was functionally prevented in rats colonised with the $L$ casei strain as translocation of bacteria to MLN was significantly reduced in the test group.

Previous studies in animal models have also shown beneficial effects of lactic acid bacteria on intestinal inflammation. Mice deficient in the interleukin 10 gene spontaneously develop colitis. In the neonatal period, these knockout mice have decreased counts of lactobacilli in the colon and an increased number of bacteria adherent or even invading the mucosa. ${ }^{15}$ Rectal delivery of $L$ reuteri to these mice re-established a balanced flora, reduced the number of translocated bacteria, and attenuated the progression to colitis. ${ }^{15}$ Most published studies showing beneficial effects of lactic acid bacteria on intestinal inflammation were performed in the same animal model of spontaneous colitis in interleukin 10 knockout mice. Thus oral administration of either VSL\#3, a mixture of eight bacterial strains, $L$ plantarum, L salivarius, or Bifidobacterium infantis, was also associated with a reduction in mucosal inflammatory activity and a significant decrease in histological lesion scores. ${ }^{16-18}$ However, the same L plantarum strain, when tested in the rat TNBS model of colitis, failed to reduce lesion scores and barrier dysfunction. ${ }^{19}$ Likewise, Pavan and colleagues ${ }^{20}$ found that pretreatment with either a L plantarum or a $L$ lactis strain had no effect on macroscopic or histological scores in mice with TNBS induced colitis. However, the number of enteric bacteria that translocated to MLN was greatly reduced in mice pretreated with any of the two strains, ${ }^{20}$ suggesting a protective effect of the lactic acid bacteria on TNBS induced barrier dysfunction. Our current study using the model of controlled microbial colonisation confirms a protective effect on mucosal barrier function and also demonstrates that the $L$ casei strain can prevent mucosal injury and inflammation induced by TNBS in the rat. In our study, previous eradication of the native flora by antibiotics and the ecological characteristics of the model (exclusion form intestinal transit) allowed mucosal colonisation by the introduced $L$ casei strain. This experimental procedure may explain the difference in efficacy between the various Lactobacillus strains. In addition, strain specific properties may also account for the difference but this hypothesis needs to be investigated by testing in parallel the different strains under the same experimental setting.

The exact mechanism by which lactobacilli can protect against the development of intestinal inflammation has not yet been established. However, it is known that certain lactobacilli adhere to mucosal surfaces, inhibit the attachment of aerobic Gram negative bacteria, and enhance secretion of mucins. ${ }^{21-22}$ These properties may be instrumental in improving mucosal barrier function and decreasing permeability to macromolecules and toxins. ${ }^{23}$ Furthermore, 
some lactobacilli, including the $L$ casei strain tested here, can downregulate inflammatory responses ${ }^{89}$ and thus mitigate tissue injury due to inflammation. Recent in vivo experiments with the same strain have demonstrated that this bacteria reduces antigen specific skin inflammation by reducing the pool of cytotoxic T cells. ${ }^{24}$ The anti-inflammatory effect of lactobacilli may be mediated by metabolites secreted by the bacteria, ${ }^{25}$ by bacterial cell components, ${ }^{26}$ or even by immunostimulatory DNA motifs under certain conditions. ${ }^{27}$

In summary, in rats colonised with $L$ casei, the extent of mucosal injury, the inflammatory response, and intestinal barrier disruption that occurs after exposure to TNBS is significantly mitigated. It is known that intestinal inflammatory responses are markedly influenced by the bacterial communities that colonise the mucosal surface. Some bacteria may induce deep transmural inflammatory responses while other bacteria appear to be able to downregulate the inflammatory process. Thus a balanced mucosal microecology could restore immune homeostasis in chronic inflammatory bowel diseases.

\section{ACKNOWLEDGEMENTS}

The authors thank Mrs Montserrat Casellas, Mrs Milagros Gallart, and Mrs Carmen Alastrue for technical assistance in the analytical procedures.

This work was supported by grants from Danone Vitapole (Palaiseau, France), Instituto de Salud Carlos III (C03/02), Ministerio de Ciencia y Tecnologia (SAF 2003-05262), and Generalitat de Catalunya (RE: 2001SGR00389).

\section{Authors' affiliations}

M Llopis, M Antolín, F Guarner, J-R Malagelada, Digestive System

Research Unit, University Hospital Vall d'Hebron, Autonomous

University of Barcelona, Barcelona, Spain

A Salas, Department of Pathology, Hospital Mutua de Terrassa,

Terrassa, Spain

Conflict of interest: None declared.

\section{REFERENCES}

1 Morris GP, Beck PL, Herridge MS, et al. Hapten-induced model of chronic inflammation and ulceration in the rat colon. Gastroenterology 1989:96:795-803.

2 Yamada T, Marshall S, Specian RD, et al. A comparative analysis of two models of colitis in rats. Gastroenterology 1992;102:1524-34.

3 Okayasu I, Hatakeyama S, Yamada M, et al. A novel method in the induction of reliable experimental acute and chronic ulcerative colitis in mice. Gastroenterology 1990;98:694-702.

4 Little JR, Eisen HN. Preparation and characterization of antibodies specific for the 2,4,6-trinitrophenyl group. Biochemistry 1966;5:3385-95.

5 Kunin S, Gallily R. Recognition and lysis of altered self cells by macrophages, a modification of target cells by 2,4,6-trinitrobenzene sulfonic acid. Immunology 1983;121:1711-18.
6 Tatsumi Y, Lichtenberger LM. Molecular association of trinitrobenzenesulfonic acid and surface phospholipids in the development of colitis in rats. Gastroenterology 1996;110:780-9.

7 García-Lafuente A, Antolín M, Guarner F, et al. Incrimination of anaerobic bacteria in the induction of experimental colitis. Am J Physiol 1997;272:G10-1.

8 Borruel N, Carol M, Casellas F, et al. Increased mucosal TNF $\alpha$ production in Crohn's disease can be downregulated ex vivo by probiotic bacteria. Gut 2002;51:659-64.

9 Borruel N, Casellas F, Antolín M, et al. Effects of nonpathogenic bacteria on cytokine secretion by human intestinal mucosa. Am J Gastroenterol 2003;98:865-70.

10 Nicklas W, Baneux P, Boot R, et al. Recommendations for the health monitoring of rodent and rabbit colonies in breeding and experimental units. Lab Anim 2002;36:20-42.

11 Morton DB, Griffiths PHM. Guidelines on the recognition of pain, distress and discomfort in experimental animals and an hypothesis for assessment. Veterinary Record April 20, 1985. For reference No 11, do you have any more details? Volume number? Page numbers?

12 Favia R, Willen R, Ar'Rajab A, et al. Acetic acid-induced colitis in the rat: a reproducible experimental model for acute ulcerative colitis. Eur Surg Res 1992;24:211-25.

13 Mainous MR, Tso P, Berg RD, et al. Studies of the route, magnitude, and time course of bacterial translocation in a model of systemic inflammation. Arch Surg 1991; 126:33-7.

14 Bezkorovainy A. Probiotics: determinants of survival and growth in the gut. Am J Clin Nutr 2001;73(suppl):399-405S.

15 Madsen KL, Doyle JS, Jewell LD, et al. Lactobacillus species prevents colitis in interleukin 10 gene-deficient mice. Gastroenterology 1999;1 16:1 107-14.

16 Madsen K, Cornish A, Soper P, et al. Probiotic bacteria enhance murine and human intestinal epithelial barrier function. Gastroenterology 2001;121:580-91.

17 Schultz M, Veltkamp C, Dieleman LA, et al. Lactobacillus plantarum 299V in the treatment and prevention of spontaneous colitis in interleukin-10-deficient mice. Inflamm Bowel Dis 2002;8:71-80.

18 McCarthy J, O'Mahony L, O'Callaghan L, et al. Double blind, placebo controlled trial of two probiotic strains in interleukin-10 knockout mice and mechanistic link with cytokine balance. Gut 2003;52:975-80.

19 Kennedy RJ, Hoper M, Deodhar K, et al. Probiotic therapy fails to improve gut permeability in a hapten model of colitis. Scand J Gastroenterol 2000;35:1266-71.

20 Pavan S, Desreumaux P, Mercenier A. Use of mouse models to evaluate the persistence, safety, and immune modulation capacities of lactic acid bacteria. Clin Diagn Lab Immunol 2003;10:696-701.

21 Bernet MF, Brassart D, Neeser JR, et al. Lactobacillus acidophilus LA1 binds to cultured human intestinal cell lines and inhibits cell attachment and cell invasion by enterovirulent bacteria. Gut 1994;35:483-9.

22 Mack DR, Michail S, Wei S, et al. Probiotics inhibit enteropathogenic E. coli adherence in vitro by inducing intestinal mucin gene expression. Am J Physiol 1999;276:G941-50.

23 García-Lafuente A, Antolín M, Guarner F, et al. Modulation of colonic barrier function by the composition of the commensal flora in the rat. Gut 2001;48:503-7.

24 Chapat L, Chemin K, Dubois B, et al. Lactobacillus casei reduces CD8+ T cellmediated skin inflammation. Eur J Immunol 2004;34:2520-8.

25 Menard S, Candalh C, Bambou JC, et al. Lactic acid bacteria secrete metabolites retaining anti-inflammatory properties after intestinal transport. Gut 2004:53:821-8.

26 Sheil B, McCarthy J, O'Mahony L, et al. Is the mucosal route of administration essential for probiotic function? Subcutaneous administration is associated with attenuation of murine colitis and arthritis. Gut 2004;53:694-700.

27 Obermeier F, Dunger N, Strauch UG, et al. Contrasting activity of cytosinguanosin dinucleotide oligonucleotides in mice with experimental colitis. Clin Exp Immunol 2003;134:217-24. 